\title{
Anion layering and steric hydration repulsion on positively charged surfaces in aqueous electrolytes
}

\author{
Qingyun $\mathrm{Hu}^{1}$, Christian Weber ${ }^{3}$, Hsiu-Wei Cheng ${ }^{1,3}$, Frank Uwe Renner ${ }^{1,2}$ and Markus Valtiner ${ }^{1,3^{*}}$ \\ ${ }^{1}$ Department for Interface Chemistry and Surface Engineering, Max Planck Institut für \\ Eisenforschung GmbH, D-40237, Düsseldorf, Germany \\ ${ }^{2}$ Institute of Materials Research (IMO), Hasselt University, Wetenschapspark 1, B-3590 Diepenbeek, \\ Belgium. E-mail: frank.renner@uhasselt.be \\ ${ }^{3}$ Institut für Physikalische Chemie der TU Bergakademie Freiberg, 09599, Freiberg
}

Keywords: Hydration forces, anions, water layering, mica, AFM

Corresponding authors*: Q. Hu (q.hu@mpie.de) and M. Valtiner (valtiner@mpie.de)

\begin{abstract}
.
The molecular structure at charged solid/liquid interfaces is vital for many chemical or electrochemical processes, such as adhesion, catalysis or the stability of colloidal dispersions. How cations influence structural hydration forces and interactions across negatively charged surfaces have been studied in great detail. However, how anions influence structural hydration forces on positively charged surfaces is much less understood. Here we report force versus distance profiles on freshly cleaved mica using Atomic Force Microscopy with silicon tips. We characterize steric anion hydration forces for a set of common anions $\left(\mathrm{Cl}^{-}, \mathrm{ClO}_{4}^{-}, \mathrm{NO}_{3}{ }^{-}, \mathrm{SO}_{4}{ }^{2-}\right.$ and $\left.\mathrm{PO}_{4}{ }^{3-}\right)$ at $\mathrm{pH} \sim 1$ in pure acids, where protons are the co ions. Solutions containing anions with low hydration energies exhibit repulsive structural hydration forces, indicating significant ion and/or water structuring within the first 1-2 $\mathrm{nm}$ on a positively charged surface. We attribute this to specific adsorption effects within the Stern layer. In contrast, ions with high hydration energies show exponentially repulsive hydration forces, indicating less degree of structuring within the Stern layer. The presented data demonstrates that anion hydration forces in the inner double layer are comparable to cation hydration forces, and that they qualitatively correlate with hydration free energies. This work may contribute to understanding interaction processes where positive charge is screened by anions within an electrolyte.
\end{abstract}




\section{INTRODUCTION}

Electric Double Layers (EDL) form when electrolyte components adsorb on a charged surface in an electrolyte due to an attraction of oppositely charged ions and alignment of dipolar molecules, resulting in a screening of the surface charge/potential. EDL thicknesses are typically on the order of about 2-3 times the Debye screening length of the electrolyte, and ions can specifically structure on the nanometer or molecular scale in the close vicinity of the solid/liquid interface. EDL structures are not only determined by the surface charge density but also by the ion properties such as their radii and hydrated structures as well as hydration energies. As a result of the EDL formation, properties of the liquid such as viscosity ${ }^{1}$, diffusion coefficient ${ }^{2}$, structuring ${ }^{3}$ at the interface differ significantly from bulk values. A detailed understanding of the molecular structure of EDLs is essential for many chemical and engineering processes, such as adsorption ${ }^{4}$, adhesion $^{5}$, catalysis ${ }^{6}$, electrochemical processes $^{7}$, clay swelling ${ }^{8,9}$, colloidal dispersions ${ }^{10,11}$, or the biomedical sciences ${ }^{12,13}$.

The mean field Poisson-Boltzmann (PB) theory ${ }^{14}$ is widely applied to describe the local charge distribution perpendicular to a charged surface in solution concentrations up to $100 \mathrm{mM}$. Above 100 $\mathrm{mM}$ i.e. at Debye lengths that are smaller than $0.96 \mathrm{~nm}$, the EDL thickness reduced less than 2-3 nm. At these dimensions the continuum based description of EDL tends to break down and unexpected trends can be observed ${ }^{15,16,17} 18$. For small dimensions, density functional theory and Molecular Dynamics (MD) simulations can provide molecular insight into the distributions of atoms and molecules, making it a common method to analyze the interfacial molecular structures. Although, there are inherent difficulties regarding how water is simulated and how surface charge or potentials are introduced into a simulation. In general, simulations seem to suggest that the structure and dynamics of water well beyond the first hydration shells are significantly affected by the presence of the ions in the vicinity of a charged surface ${ }^{19,20,21}$ although some ${ }^{22,23}$ disagree on that. The broadly studied systems with MD methods are alkali metal or alkaline earth metal cations, but rarely anions have been studied ${ }^{24,25}$.

Force microscopy experiments further revealed how structuring at solid interfaces leads to what is now referred to as steric hydration forces and oscillatory forces ${ }^{26}$. When two surfaces or particles approach closer than a few nanometers, the continuum DLVO theory ${ }^{27}$, attractive van der Waals forces added with repulsive electric double layer forces, fails to describe the interactions, because molecular sizes and shapes of solvent and ions become critical ${ }^{28}$. As a result, additional hydration forces originate from the geometry or structure of molecules and ions in the confined vicinity in aqueous solution. The magnitude and distance dependent profile of hydration forces depend on the liquid medium and the surface structure. Short range hydration forces are particularly important for adhesion and lubrication. It was also demonstrated that protons do not cause significant hydration repulsions ${ }^{29,30}$.

Cation structuring at negatively charged surfaces was studied in much greater detail compared to anion structuring ${ }^{2,31}$. For example, recently Bourg and Fenter ${ }^{32}$ combined XRR measurement and MD simulations and reported the Stern layer structure and energetics of alkali chloride solutions on mica 
surfaces. They observed two adsorption types of inner-sphere surface complexes, and pointed out that the first layer is structured water adsorbed to the surface, while the second layer was largely impacted by adsorbed ions. How anions structure at interfaces was just recently studied on metal surfaces ${ }^{33}$ and to the best of our knowledge no studies focused on characterizing anion hydration forces in the absence of metal co-ions.

Here, we provide a qualitative understanding of structural forces due to anion adsorption in the simplest system with only anions existing in the nano-scale vicinity at an interface, while co-ions are exclusively protons that do not show any structural forces at an interface ${ }^{34}$. Specifically, we perform a series of force distance measurements at $\mathrm{pH} \sim 1$ using different acids on mica surfaces. As shown in Figure 1, we use Atomic Force Microscopy (AFM) to discuss qualitative information about the charge distribution at the solid electrolyte interface through evaluation of the interaction forces between two opposing double layers. From the variation of the interaction force profiles, we discuss mechanisms of anion structuring at positively charged interfaces in aqueous systems. Interestingly we find pronounced specific effects of anions on hydration forces. Our data suggests that significant hydration forces, which are qualitatively comparable to cation hydration forces, arise from anion adsorption at positively charged surfaces.

\section{MATERIALS AND METHODS}

Chemicals and materials: All chemicals were purchased from Sigma-Aldrich at the highest available purity and diluted with Milli-Q water (Millipore, resistivity of $\geq 18.2 \mathrm{M} \Omega \mathrm{cm}$ and a TOC $\leq 2$ $\mathrm{ppb}$ ) to prepare the required solutions of demanded $\mathrm{pH}$ value. Then the solutions were vacuum degassed for more than two hours and purged with clean argon gas for at least one hour. The $\mathrm{pH}$ values were measured before and after degassing the solution using a $\mathrm{pH}$ meter (InoLab pH720 from WTW GmbH).

Optical grade V1 ruby-mica, with a nominal composition $\mathrm{KAl}_{2}\left(\mathrm{Si}_{3} \mathrm{Al}\right) \mathrm{O}_{10}(\mathrm{OH})_{2}$, was obtained from S\&J Trading Company (NJ, U.S.A.) as sheets of about $20 \times 20 \mathrm{~cm}$ surface area. The mica was cleaved on both sides under the laminar flow hood to provide an atomically smooth hydrophilic surface. The sheet is tailored into $25 \times 25 \mathrm{~mm}$ square with a thickness normally around $2 \mathrm{~mm}$. Afterwards it was blown with clean argon gas and immediately mounted onto the AFM fluid cell, followed by injection of the filtered solution to avoid the contamination of the mica surface. Before the measurement, the system was equilibrated for about 2 hours to complete the ion exchange and minimize thermal drift.

Atomic Force Microscopy (AFM): In this work, a commercial AFM instrument (JPK Nanowizard), was used to perform conventional force measurements. The AFM is placed on an antivibrational table in a noise-isolated box. Si cantilevers and tips, with surfaces that are covered with $\mathrm{SiO}_{2}$ of around 1-3 nm thickness due to air exposure ${ }^{35}$, were chosen as tip material. Contact mode probes with rectangular silicon cantilevers (Nanosensors, PPP-CONTR) with a spring constant $0.1 \sim$ $0.2 \mathrm{~N} / \mathrm{m}$ were used. Before use the probes were thoroughly cleaned and oxidized by sulfuric acid, 
followed by extensive rinsing in deionized water and ethanol and drying in a stream of argon gas. The fluid cell was made from PEEK providing a chemical inert environment for all the solutions. All experiments were conducted in ambient lab temperature around $25{ }^{\circ} \mathrm{C}$ and ambient atmosphere $(1$ bar). We used an approaching velocity of $0.05 \mu \mathrm{m} / \mathrm{s}$. It has to be stressed, that AFM does not measure the actual distance between a tip and the surface. Instead, the zero distance in AFM is calibrated using the linear extension of the AFM cantilever in hard contact, where no deviations from this linearity are observed (generally above $5 \mathrm{nN}$ applied normal force).

Three force curves were acquired at 100 different locations, which were equally spaced from each other over a $100 \mu \mathrm{m}^{2}$ area. A schematic of the experimental system used is shown in Figure 1. The Stern layer is comprised of Inner Helmholtz Plane (IHP) and Outer Helmholtz Plane (OHP), beyond OHP, the diffuse layer, where the typical decay length is known as the Debye length, has its plane of origin. The inset on the right shows a schematic of the expected ion distribution near the surface in acids at $\mathrm{pH} \sim 1$. Protonation charges the surface positively, and anions accumulate within stern layer leading to distinct layering.

Data modeling: The Si tips used have conical shapes with radii $\mathrm{R}$ ranging from 5-50nm, with a typical half cone angle around $19^{\circ}$ (manufacturer information). Here, we use a conical shape tip with a sphere end on plate geometry to model the measured interaction forces. The Derjaguin-LandauVerwey-Overbeek theory (DLVO theory) ${ }^{27}$, which formulates the interaction forces between two surfaces as the superposition of Van der Waals force and diffuse EDL forces, is applied in our fitting. We additionally describe EDL forces $F_{E D L}$ as a linear superposition of both (1) diffuse layer forces $\mathrm{F}_{\mathrm{DL}}$, described by the Gouy-Chapman theory ${ }^{36}$, and (2) we include hydration forces within Stern layer $F_{\text {HYD }}$ at distances below about 1-2 nm, which takes into account of forces due to specific ion structures within the inner well-structured $\mathrm{EDL}^{37}$. Therefore, we have:

$$
\mathrm{F}(\mathrm{D})=\mathrm{F}_{\mathrm{VDW}}+\mathrm{F}_{\mathrm{EDL}}=\mathrm{F}_{\mathrm{VDW}}+\mathrm{F}_{\mathrm{DL}}+\mathrm{F}_{\mathrm{HYD}}
$$

(Equation 1)

where $\mathrm{D}$ is the apparent separation distance between the two surfaces. The Van der Waals force FvDw between an AFM probe with radius $\mathrm{R}$ and a flat surface is modelled according to Argento and French $^{38}$ :

$$
\mathrm{F}_{\mathrm{VDW}}=-\frac{2 A_{H} \mathrm{R}^{3}}{3(2 \mathrm{R}+(\mathrm{D}+\mathrm{D} 0))^{2}(\mathrm{D}+\mathrm{D} 0)^{2}}
$$

where $A_{H}$ is the effective Hamaker constant and $D_{0}$ characterizes a shift of the plane of origin. We use a Hamaker constant of $3.5 \times 10^{-20} \mathrm{~J}^{39}$ for the Si-Water-Mica system, with shift of the plane of origin of 1 $\mathrm{nm}$, which corresponds to the oxide thickness. Shifting the onset of VDW force is mathematically very similar to more complex models that take into account the thin oxide film ${ }^{40,41}$. Here we use a shift of the onset in order to avoid complex fittings of the VDW forces, which are overpowered by hydration forces in all the presented measurements. Hence, using more complex models would not change the results significantly. 
A monotonic exponential hydration force arising from the presence of ordered solvent layers (Stern layer) can be described by an empirical expression for the steric hydration force ${ }^{42}$ as shown in Equation 3. Since the hydration forces dominate in the region where $\mathrm{D}<<\mathrm{R}$, we can use Derjaguin's approximation to calculate the interaction forces from the proposed empirical interaction potential for hydration forces.

$$
\mathrm{F}_{\mathrm{HYD}}=2 \pi R W(\mathrm{D})=2 \pi R W_{0} \cdot \mathrm{e}^{-\frac{\mathrm{D}}{\lambda_{\mathrm{H}}}}
$$

Where the $\mathrm{W}_{0}$ is the prefactor of the interaction hydration energy, $\lambda_{\mathrm{H}}$ denotes the hydration force decay length. The physical meaning of these two factors should be closely correlated to the origin of the hydration force. We want to emphasize that still no physical model for the derivation of the pre-factor $\mathrm{W}_{0}$ exists. Also, in AFM it is not clear how $\mathrm{W}_{0}$ and the radius (which is unknown) can be separated; as such we quote the entire pre-factor rather than single parameters in all tables below and only interpret relative trends. In the fitting presented below, two hydration force terms are sufficient to fit the data.

The diffuse part of the double layer repulsion is not necessary for fitting the data measured in high concentration acids. Hence $F_{D L}=0$ and the full equation to fit an (1) inner and (2) an outer Helmholtz plane in acidic solution at $\mathrm{pH}=1$ is as follows:

$$
\mathrm{F}(\mathrm{D})=\mathrm{F}_{\mathrm{VDW}}+2 \pi R W_{01} \cdot e^{-\frac{D}{\lambda_{H 1}}}+2 \pi R W_{02} \cdot e^{-\frac{D}{\lambda_{H 2}}}
$$

(Equation 4)

Only for experiments in Milli-Q water, an electric double layer contribution $\mathrm{F}_{\mathrm{DL}}$ had to be included. Here, we used a constant charge boundary condition solved by H.J. Butt ${ }^{43}$ for the analytical estimation of EDL forces for AFM tips on a flat surface, and added the expression to Equation 4.

\section{RESULTS}

In this work we characterize interaction forces. Specifically, hydration forces measured between mica surfaces and AFM tips in $\mathrm{pH} \sim 1$ electrolyte and compare them with pure Milli-Q water at $\mathrm{pH} \sim$ 5.5. Although electric double layers in principle form on the tip as well as on the surface, according to Martin-Jimenez et al. ${ }^{44}$ the nanoscale roughness of the tip does not contribute any ordered layer compared to atomically smooth mica surfaces. Therefore, we restrict the interpretation of layering on the mica surface, with little distortion originating from the tip. Varying acids and hence anions allows us to characterize the effect of different sizes, valences and hydration enthalpies on the interfacial structure formation and on anion hydration forces on mica surface.

We first characterize interfacial structuring of water, and revisit water structuring measurements in Milli-Q water, and then introduce anions by lowering the $\mathrm{pH}$ with $\sim 100 \mathrm{mM}$ acids. We assume, and also demonstrate, that this allows us to reverse the charge on the mica surfaces, introducing an anion dominated double layer without any accompanying metal cations since there is only protons present as co-ions. Protons are known to not exhibit measureable hydration forces ${ }^{26,29,31}$.

Interaction forces in water. Figure 2 shows a force versus distance profile (F/D) recorded in Milli-Q water $(\mathrm{pH} \sim 5.5)$ as a function of the tip sample separation distance $\mathrm{D}$ during approach of an 
AFM tip and a mica surface. Figure 2(a) displays a single typical F/D profile. Throughout this work, the zero separation distance $\mathrm{D}=0$ was defined as the point where the tip comes into an apparent hard contact with the surface. At this point applying higher forces results in cantilever bending proportional to the piezo movement, assuming zero deformation of surface and tip. In pure water, after the desorption of the surface $\mathrm{K}^{+}$ions from mica in solution ${ }^{45}$, the remaining mica surface is effectively a hard wall defined by the densely packed basal-plane oxygen atoms, augmented with an array of ditriogonal cavities ${ }^{35}$.

In typical individual force versus distance curves shown in Figure 2(a) at $\mathrm{D}<2.5 \AA$ a steep exponential rise of the force, followed by a clear kink at $\mathrm{D} \sim 2.5 \AA$ was observed, along with a double exponential diffuse layering at larger distances. We can separate the curve into three domains: (1) $\mathrm{D}<$ $2.5 \AA$; (2) $2.5 \AA<\mathrm{D}<1 \mathrm{~nm}$; and (3) D $>1 \mathrm{~nm}$. At D $>1 \mathrm{~nm}$, the data can be well fitted with conventional DLVO theory with a Debye length of $38 \mathrm{~nm}$ and a diffuse double layer charge of $|0.013|$ $\mathrm{C} / \mathrm{m}^{2}$ at the plane of origin at $\mathrm{D} \sim 1.2 \mathrm{~nm}$. The measured Debye length of $38 \mathrm{~nm}$ is smaller than the expected $50 \mathrm{~nm}$ for $\mathrm{pH} 5.5$ Milli-Q water. This is either due to the lower force sensitivity of AFM at large distances. As a consequence, the detection of a zero force regime at $\mathrm{D}>60 \sim 80 \mathrm{~nm}$ may be lower than the measured Debye length artificially. Other possibilities are, that the analytical equation is not applicable, or that impurities in the clean water such as additionally dissolved airborne species such $\mathrm{CO}_{2}$. At $2.5 \AA<\mathrm{D}<1 \mathrm{~nm}$ an exponential steric hydration force with a decay length of $\lambda_{\mathrm{H}}=$ $3.35 \AA$ was observed. Further, in the regime at $\mathrm{D}<2.5 \AA$, the additional steric hydration can be fitted with an additional exponential repulsion term with a steep slope of $1.8 \AA$ as shown by the blue solid line, which may indicate the last strongly bound water layer to the surface.

Figure 2(b) depicts a dynamic 2-dimensional (2-D) force versus distance map averaged over more than 50 measurements distributed at random locations. Here the y-axis is linear and the statistical color scale is labeled on the top of the plot, indicating features where about two thirds of all recorded force traces are observed. The inset shows a zoom-in for the last $5 \mathrm{~nm}$, together with a histogram that condenses the force counts into a distance dependent layering density profile. This layering density profiles provides additional statistical information about the average structural forces picked up during compression.

Figure 2(b) again suggests the same distinct regimes with a long-range diffuse interaction force, that decays into thermal noise at $\mathrm{D} \sim 60-80 \mathrm{~nm}$, indicative of the force detection limit of at about 20$30 \mathrm{pN}$. At smaller separation distances $\mathrm{D}<1 \mathrm{~nm}$, the density plot shows that the force distance trace becomes less defined with only $80-90 \%$ of the force traces following exactly the main force trace during pushing through the exponentially rising hydration layer. This suggests that this layer is diffusely structured extending over about $\Delta \mathrm{D} \sim 6-8 \AA$ in total. At $\mathrm{D}<2.5 \AA$ we do find that force profiles show much less variation again, while the specific instability observed in individual curves appears statistically smeared out at various applied forces and hence distances. However, the center of the Gaussian fits shown in the histogram, provides a measure for where these jump-in events occur most likely. The histogram shows three distinct peaks below $\mathrm{D}<1 \mathrm{~nm}$, which are a first rather broad 
distribution centered at $\mathrm{D} \sim 6.8 \AA$ with a variance of $1.7 \AA$ that characterizes the outer diffuse structuring; and at $\mathrm{D} \sim 2.5 \pm 0.8 \AA$ and at $\mathrm{D} \sim 0.9 \pm 0.4 \AA$ we find two more populations that indicate the water layering in the close vicinity of the surface.

This data fits well with a molecular surface layer of water with oxygens of water pointing upwards (see schematic in Figure 3). This interpretation is in line with work by Toney et al. ${ }^{46}$ who found oxygen-up or down average orientation for negative or positive charge, respectively. Such molecular arrangements naturally result in steric forces. Following this model, the layer at $\mathrm{D} \sim 2.54 \AA$ may resemble a second layer structure of the water network with the protons connect to the oxygen of the first water layer with hydrogen bonds. This result compares indeed well with previous work by other groups. With high resolution X-Ray Reflectivity (XRR) Cheng et al. ${ }^{35}$ revealed, for example, interfacial water ordering with spacing of $2.5 \sim 2.7 \AA$. Also Monte Carlo simulation work done by Park et al. ${ }^{47}$ supported that the water molecule is bound closely to the ditrigonal cavities on the mica surface with a distance about $2.5 \AA$ to the surface oxygen in the nominal plane direction. Furthermore, with Molecular Dynamics (MD) simulation, Xu et al. ${ }^{48}$ showed that the first water concentration peak splits into two with an increasing surface charge density from 0 to $-0.2034 \mathrm{C} / \mathrm{m}^{2}$. Similar to this work, three distinct layers of water were also observed by Qiu et al. ${ }^{49}$. They found that the adsorbed water layer splits into three layers and the first layer locates much closer to the surface if the surface charge density increases to $-0.3 \mathrm{C} / \mathrm{m}^{2}$. Based on our force distance profiles and the histogram plot, in Figure 3 we provide a schematic of the water structure on mica at $\mathrm{pH} \sim 5.5$ with no salt added $\left(\mathrm{CO}_{2}\right.$ from the air lowers the $\mathrm{pH}$ ), characterizing the inner two layers as strongly bound IHP, the third more diffuse appearing layer as an OHP and a diffuse layer. While the water network seems to provide a stiff structural network close to the surface, the proton distribution in this network is not a direct measure. Still, the calculated diffuse double layer potential at the OHP, where the diffuse EDL has its plane of origin is $\sim|210| \mathrm{mV}$ using Grahame's equation and the fitted diffuse layer charge. Assuming a mica surface potential off $\sim 400 \mathrm{mV}$ for a fully dissociated surface, this means that the surface potential is screened by protons down to $50 \%$ in the stern layer, where water forms a more rigid network.

It is now interesting to compare this data to earlier work by Israelachvili et al. ${ }^{39}$ who observed, using a surface force apparatus (SFA), for the first time oscillatory hydration forces with a periodicity of $2.5 \pm$ $0.3 \AA$. In contrast to SFA data, we find no strongly pronounced periodicity beyond the first layer of water, but an increasingly unordered structure away from the mica interface. A very strongly bound inner layer extends into consecutively less strongly bound outer layers with increasing distance from the charged surface. Beyond the first layer, we find a spacing that is consistent with SFA. The first layer is located at $2.57 \AA$ corresponding to the molecular dimensions of water. In contrast, the structure beyond this layer is less ordered with an exponential increase of the interaction force over a distance of 5-6 $\AA$ which is about two times the water dimensions, comparing well to SFA data. Compared to SFA, AFM F-D profiles do not impose a large scale confinement during interaction. As such, it seems that AFM data captures the layering on non-confined mica as probed by a hydrated $\mathrm{Si}$ tip. Taken with a grain of salt, our data suggest that water does structure distinctly at mica surfaces as 
previously argued, but with increasing thermal disorder into the bulk. One interesting additional detail compared to SFA data is, that the innermost layer compressed with a slope of $1.7 \AA$. This may be a layer of water lying down flat, that could not be forced out from contact at pressures that can be achieved with SFA. With its small probe radius, the applied pressure in AFM experiments can be $10^{3} \sim 10^{5}$ orders of magnitude higher, considering typical maximum loading forces of $5 \mathrm{nN}$ and $10 \sim$ $100 \mathrm{mN} / \mathrm{m}$, as well as the typical contact areas of $25 \mathrm{~nm}^{2}$ versus $0.0078 \mathrm{~mm}^{2}$ for AFM and SFA, respectively. It is however more likely that the AFM tip may simply impose a final compression layer that differs compared to mica/mica experiments in SFA.

Interaction forces in various acids at $\mathbf{p H}=1$. Once freshly cleaved mica is exposed to solution, the potassium ions hydrate and leave the mica surface with a negative surface charge. Due to protonation of the negative surface sites the mica surface becomes uncharged at $\mathrm{pH}=2 \sim 3$. At even lower $\mathrm{pH}$ when protons or hydroniums are in excess, over adsorption of protons may result in an effective positive charging of the surface, because the exposed oxygens may protonate as following reaction, at the surface or even within the octahedral aluminum layer of mica:

$$
-\mathrm{SiO}+\mathrm{H}_{3} \mathrm{O}^{+} \leftrightharpoons-\mathrm{SiOH}^{+}+\mathrm{H}_{2} \mathrm{O}
$$

There is however no literature that proves or disproves that mica indeed charges overall positive at low $\mathrm{pH}$ due to protonation. Below, it will become clear that anion adsorption does indeed occur at these low $\mathrm{pH}$ values, indicating positive surface charging by protonation.

In particular, we use different acids at $\mathrm{pH} \sim 1$. This leaves us with only anions and protons as coions in the electrolyte. Protons, as opposed to metal cations as charged co-ions, show no distinct hydration forces ${ }^{26}$. This enables a direct measure of specific anion hydration effects on mica without interference of metal cations. Ion properties relevant to our discussion below are listed in Table $\mathbf{1}$. RMS roughness of less than $5 \AA$ recorded before and after experiment indicate that the mica surface is not attacked by the acidic solution. Also, in AFM no holes or other defects indicating an acidic attack were detected (data not shown). This is in line with expectations, that the mica basal plane is at the experimental conditions resistant to dissolution, and preferable etching proceeds via other low index planes that expose the octahedral aluminum layer ${ }^{50}$.

Figure 4 shows 2-D force versus distance characteristics of an average of over 50 individual force runs recorded between mica and a silica tip in (a) $\mathrm{HCl}$, (b) $\mathrm{HClO}_{4}$, (c) $\mathrm{HNO}_{3}$, (d) $\mathrm{H}_{2} \mathrm{SO}_{4}$ and (e) $\mathrm{H}_{3} \mathrm{PO}_{4}$. Data was fit using Equation 4, and representative individual comparison of fit and data are shown in Figure 5. Relevant fit parameters according to Equation 4 obtained from fitting individual force curves are listed in Table 2. The fitted decay lengths are very accurate and independent of the tip radius, while the exponential pre-factors of the hydration repulsions in Equation $\mathbf{4}$ are not decoupled from the fitted and approximately known tip radius. As such, we restrict our discussion of fitting parameters to the decay lengths. 
Figure 4(a) shows recorded F-D curves in $\mathrm{HCl}$, which clearly show strong structural hydration forces that are likely related to specific double layer structuring of $\mathrm{Cl}^{-}$ions. In total, we find two pronounced instabilities. The first instability is a very clearly pronounced jump-in from $\mathrm{D} \sim 7 \AA$ to about $\mathrm{D} \sim 3 \AA$. This jump-in distance compares to $\sim 2 / 3$ of the hydrated ion radius, shown in Table 1. At $\mathrm{D} \sim 3 \AA$ a very distinct steep exponential rise of the force with a decay length of $2.5 \AA$ appears, implying compression of a strongly bound layer of hydration water. In detail, after exponential compression over $2 \AA$ a sudden structural jump over $1 \AA$ into the hard wall at $\mathrm{D}=0$ can be observed, suggesting a push-out of a compressible structured layer.

First, this data indicates strong layering of hydrated $\mathrm{Cl}^{-}$ions forming the IHP below D 8-10 $\AA$, as well as structural water layering at compressions to distances below $3 \AA$. In addition to that, an outer disordered layer extends from $\mathrm{D} \sim 8-10 \AA$. This layer can be fitted well with a hydration repulsion decay length of $\sim 6 \AA$; no diffuse electric double layer interaction is needed for fitting the data. Specifically, the expected Debye length of $\sim 9.6 \AA$ does not fit the observed exponential repulsion, which suggests that this long range contribution is actually an additional hydration repulsion originating from the OHP. In summary, the surface charge is fully screened by a strongly structured Stern layer with a distinct IHP that compresses over the distance of $3 \AA$, an OHP with specifically bound chlorides at a distance of about 4-10 $\AA$, and a diffuse OHP with disordered chlorides that extends to $20 \AA$.

What does this result imply for the mica charge itself? Clearly, this result is not compatible with a negatively charged or uncharged mica surface at $\mathrm{pH}=1$. On a negatively charged surface, layering should be comparable to the pure water case, which it is clearly not. Anions would not remain in the close vicinity. Also, adsorption of chlorides onto uncharged mica would result in a net negative over charging of the surface, which in turn results in a diffuse proton layer that screens this charge over the Debye length, this is not observed either. This data hence confirms a positive charging of mica at $\mathrm{pH}$ 1 , specific anion adsorption and strong anion hydration forces below $\mathrm{pH} \sim 2-3$, which is the isoelectric point. ${ }^{51}$

Figure 4 (b) shows force distance profiles recorded in perchlorate acid at $\mathrm{pH} \sim 1$. Here, we observe only one pronounced structural jump-in from $\mathrm{D} \sim 10 \AA$ to about $\mathrm{D} \sim 6 \AA$. This jump-in distance of $\sim 4 \AA$ again amounts to $\sim 2 / 3$ of the hydrated ion diameter, shown in Table 1. Again, this indicates a specifically adsorbed layer of anions that is expelled under compression. At distances below $6 \AA$ we observe a strong exponential repulsion with a decay length of $2.5 \AA$, which is again close to the molecular water diameter. The absence of structural features in this layer may imply compression of a disordered layer of hydration water. Interestingly, compared to $\mathrm{HCl}$, a considerably longer ranged exponential repulsion with a decay length of $13.5 \AA$ extends from $10 \AA$ to about 50-60 $\AA$. This decay length exceeds the expected Debye length of $9.6 \AA$. An interfacial activity coefficient of 0.7, which could explain an increased Debye length, seems unreasonable at an ionic strength of $0.1 \mathrm{M}$, hence this decay length should likely be interpreted as a considerably longer ranged hydration 
repulsion. This suggests that $\mathrm{ClO}_{4}^{-}$ions do not specifically adsorb onto mica, but they form a fairly extended Helmholtz layer with a high ion concentration in the inner Helmholtz plane, as indicated by the observed structural force.

Figure 4 (c) shows force distance characteristics recorded in nitric acid at $\mathrm{pH} \sim 0.97$. Interestingly, here the statistic forces versus distance characteristics show two layering structures that appear equally likely at $\mathrm{D}<1 \mathrm{~nm}$. Since we are averaging over 50 measurements recorded at different locations, this suggests a heterogeneous inner double layer structure over the entire surface area. As can be seen in Figure 5, we find a set of curves that shows an exponential rising repulsion from $10 \AA$ with a decay length of $2.5 \AA$ followed by a clear structural jump from $\mathrm{D} \sim 5 \AA$ into the hard wall at $\mathrm{D}=0$. Considering the asymmetry of the planar nitrate ion, this jump compares well to the "equatorial diameter" of the hydrated nitrate ion, or to $\sim 2 / 3$ of the axial diameter. The second set of curves shows an exponentially rising repulsion starting at smaller distances of $\mathrm{D} \sim 6-8 \AA$ with a similar decay length of $\sim 2 \AA$. This repulsion shows no structural features, indicating a compression of disordered hydration water. Also in nitric acid solution no long ranged electric double layer force was observed, indicating a considerable charge screening due to the existence of a compact anion layer in the Helmholtz plane with specifically adsorbed nitrate ions.

Figure 4 (d) and (e) show the effect of multivalent sulfate and phosphate anions. These multivalent anions differ from the monovalent anions not only due to higher charge but also their dissociation characteristics. Due to different protonation equilibria, also multiple protonated species of ions may exist in solution, which has to be taken into account for calculating and comparing to the Debye length. In sulfuric acid there are both $\mathrm{HSO}_{4}{ }^{-}$and $\mathrm{SO}_{4}{ }^{2-}$ ions with a ratio of approximately $2: 1$, while in phosphoric acid the prevalent anion is $\mathrm{H}_{2} \mathrm{PO}_{4}^{-}$. We can see that those two anion F-D profiles share three common features:

(1) At $\mathrm{D}<5 \AA$ an exponential force with a decay length of $2.5 \AA$ overpowers a longer ranged exponential repulsion. The inner layer decay compares well to the molecular water size, indicating again that dehydration of disordered surface bound water may be the main compression mechanism in this regime.

(2) At $\mathrm{D}>1 \mathrm{~nm}$, exponentially diffuse layers with decay lengths of $5 \AA$ and $7 \AA$ for the phosphate and sulfate respectively can again not be described by the expected Debye length. No structural features can be observed. In contrast to the other ions, this indicates a thermally disordered anion layer with non-specifically adsorbed anions. Interestingly, the decay lengths compare reasonably well to the hydrated ion radii given in Table 1.

(3) In the individual curves, the phosphate force distance characteristics indicate a $2.5 \AA$ structural breakdown, which we attribute to a water layer. In the statistical analysis shown in Figure 4, this jump distance is smeared out, indicating a quite disordered structure.

\section{DISCUSSION}


In summary, we find a consistent qualitative scenario for interaction force profiles in high concentration acids of $\mathrm{pH} \sim 1$ with varying anions. General qualitative features of the observed force profiles are the following.

First, all force profiles are dominated by hydration effects. No electric double layer charging was observed within the sensitivity of AFM. This indicates that the diffuse layer potentials are zero or at least less than 10-15 $\mathrm{mV}$. Second, all force profiles exhibit a short range inner hydration repulsion with a characteristic decay length of 2-2.5 $\AA$ at $\mathrm{D}<3-4 \AA$, indicating a compression of adsorbed water below 3-4 $\AA$. This compression can be diffuse or it may form structural features as seen for the chloride or phosphate. Considering the Hofmeister series ${ }^{52}$, it seems that cosmotropic ions are more likely not to disturb the interfacial layering of water close to a solvated surface and vice versa, however there is no strict dependency.

Third, all force profiles can be well described by adding a second - outer Helmholtz plane exponential hydration repulsion. This repulsion shows structural breakdowns and transition into an inner hydration repulsion at characteristic distances $\mathrm{D}_{\mathrm{T}}$ for chlorides, perchlorate and nitrate as summarized in Table 2. In contrast, phosphate and sulfate show no breakdown of the outer hydration layer with a smooth transition at $\mathrm{D}_{\text {T }}$ from outer to inner layer hydration repulsion. Interestingly, this correlates with the hydration energy of the ions quoted in Table 1. Multivalent ions with high hydration energy show no structural features. The high cost for dehydration may bind them less strongly to the surface, leading to a smooth push out of hydrated ions. In contrast, low hydration energies may allow anions to strip their hydration shell at the interface leading to specific adsorption and consequently structural forces due to hydration water breakdown.

Fourth, the characteristic hydration repulsion decay lengths $\lambda_{H}$ shown in Table 2 are generally within the range of the molecular dimension of the compressed species, which is $2.5 \AA$ for the water layer compression and 5-7 $\AA$ for disordered ion layer compressions in the outer hydration layers. The exception is perchlorate. Perchlorate exhibits an outer layer hydration repulsion that is $\sim 2$ times its diameter, and considerably larger compared to the expected Debye length. In agreement with its pronounced chaotropic behavior, this may indicate a significant water disorder in the outer hydration shells, which can effectively lower the dielectric constant. A lower dielectric constant and hence less pronounced charge screening leads to a larger average distance between like charged ions within the hydration layer. This may cause the observed "swelling" of this layer.

One aspect that remains unclear from force measurements with the AFM is, whether dehydrated ions are indeed pushed out of contact, or whether ions dehydrate and condense into the lattice during compression. AFM does not measure absolute distances ${ }^{53}$, so we cannot deduce, if the distance $\mathrm{D}=0$ is the "real" contact between the two opposing solid surfaces. Here, adhesion measurements can give some insight. Figure 6 shows that the adhesion is generally quite low in all solutions indicating a relatively low interfacial energy as expected considering Lippmann's equation for highly charged surfaces. It appears that solutions containing ions with lower hydration energy results in smaller 
adhesion. This may be interpreted as a result of ion condensation into the mica lattice during hard contact if the hydration energy is low, while the remaining layer may be a less thick proton containing water layer in case of strongly hydrated ions, which leave the contact area. This may effectively change the minimum approach distance into the adhesive minimum. As a result the adhesive energy may increase slightly due to increased VDW interactions at smaller distances. Our data can however only serve as an indicator, and other highly resolved techniques such as diffraction based analysis of the interfacial ion profile in confinement ${ }^{54,55,56}$ and non-confinement ${ }^{32}$ may provide further insight into ion distributions at interfaces.

\section{CONCLUSION}

Anions structure strongly at positively charged mica surfaces, leading to significant inner and outer hydration layer repulsions within the Stern layer in acids of $\mathrm{pH} \sim 1$. The entire surface charge of mica can be screened within a $2-3 \mathrm{~nm}$ thick hydration layer in acid solutions at $\mathrm{pH} \sim 1$. The inner hydration repulsions seem dominated by interfacial water ordering, while the outer layer hydration repulsion is dominated by anion layers. This is in good agreement with cation layering on mica, where inner-sphere surface complexes formed by the first water layer and a second layer of adsorbed cations $^{32}$ was observed.

We further find that outer layer anion hydration forces can be structural or monotonic, which is similar to hydration forces reported for cations. Ion structuring and screening properties strongly depend on the thermodynamic hydration characteristics of the anions used, while Hofmeister effects have a limited influence on the characteristic behavior. The hydration energy of anions and anion dehydration during compression may be the driving force determining both hydration repulsion and adhesion hysteresis.

Chloride, nitrate and to a lesser extend perchlorate with relatively lower hydration energies show strong specific and non-specific adsorption effects and hence structural steric hydration forces. Strongly hydrated ions such as sulfate and phosphate do not adsorb specifically hence do not structure compact layer. This results in an exponentially decaying steric hydration force without pronounced structural features, and an outer hydration layer that is purely monotonic. Still the inner water dominated hydration layer can still be structured (as observed for the phosphate).

The hydration energy of the anions offers a potential guideline for predicting interfacial ion behavior during compression of hydration layers. If the ions tend to specifically adsorb $\left(\mathrm{HCl}, \mathrm{HNO}_{3}\right)$, the hydration force is likely related to compression of hydration water of the ions resulting in structural jumps due to a condensation of dehydrated ions into the lattice. In contrast for ions having strong hydration energies $\left(\mathrm{H}_{2} \mathrm{SO}_{4}, \mathrm{H}_{3} \mathrm{PO}_{4}\right)$, the hydration force is related to pushing these ions out of contact, which is in line with the observed adhesion hysteresis.

In summary, we demonstrate that anion structuring in the inner double layer is very pronounced and that it correlates with hydration free energies. This may contribute to understanding processes where positive charge is screened by anions within an electrolyte, including corrosion science, protein folding or colloidal stability of positively charged system (e.g. amines in biologic soft matter). 


\section{ACKNOWLEDGEMENTS}

Q.H and M.V. acknowledge funding by the Deutsche Forschungsgemeinschaft (DFG) through the cluster of Excellence (EXC 1069) RESOLV at the Ruhr-University in Bochum. M.V. and C.W. acknowledge support by the European Research Council (ERC) through starting grant 677663 (CSI.interface). 


\section{REFERENCES.}

(1) Wang, X.-j.; Bai, S.-X.; Huang, P., Theoretical analysis and experimental study on the influence of electric double layer on thin film lubrication. Frontiers of Mechanical Engineering in China 2006, 1 (3), 370-373.

(2) Glaus, M. A.; Aertsens, M.; Appelo, C. A. J.; Kupcik, T.; Maes, N.; Van Laer, L.; Van Loon, L. R., Cation diffusion in the electrical double layer enhances the mass transfer rates for $\mathrm{Sr} 2+, \mathrm{Co} 2+$ and Zn2+ in compacted illite. Geochimica et Cosmochimica Acta 2015, 165, 376-388.

(3) Giera, B.; Henson, N.; Kober, E. M.; Shell, M. S.; Squires, T. M., Electric Double-Layer Structure in Primitive Model Electrolytes: Comparing Molecular Dynamics with Local-Density Approximations. Langmuir 2015, 31 (11), 3553-3562.

(4) Tinnacher, R. M.; Holmboe, M.; Tournassat, C.; Bourg, I. C.; Davis, J. A., lon adsorption and diffusion in smectite: Molecular, pore, and continuum scale views. Geochimica et Cosmochimica Acta 2016, 177, 130-149.

(5) Hilner, E.; Andersson, M. P.; Hassenkam, T.; Matthiesen, J.; Salino, P. A.; Stipp, S. L. S., The effect of ionic strength on oil adhesion in sandstone - the search for the low salinity mechanism. 2015, 5, 9933.

(6) Kovac, Z.; Sambucetti, C. J., Importance of Electrical Double Layer in Technological Applications. In Electrochemistry in Transition: From the 20th to the 21st Century, Murphy, O. J.; Srinivasan, S.; Conway, B. E., Eds. Springer US: Boston, MA, 1992; pp 39-49.

(7) Yuan, H.; Shimotani, H.; Ye, J.; Yoon, S.; Aliah, H.; Tsukazaki, A.; Kawasaki, M.; Iwasa, Y., Electrostatic and Electrochemical Nature of Liquid-Gated Electric-Double-Layer Transistors Based on Oxide Semiconductors. Journal of the American Chemical Society 2010, 132 (51), 18402-18407.

(8) Layered Mineral Structures and their Application in Advanced Technologies. Mineralogical Society of Great Britain and Ireland: 2011; p 387.

(9) Prikryl, R.; Kolariková, l.; Hanus, R., Water sorption and dilatation of bentonites and montmorillonite-rich clays. In Expansive Soils, Taylor \& Francis: 2006.

(10) Kellens, E.; Bové, H.; Conradi, M.; D’Olieslaeger, L.; Wagner, P.; Landfester, K.; Junkers, T.; Ethirajan, A., Improved Molecular Imprinting Based on Colloidal Particles Made from Miniemulsion: A Case Study on Testosterone and Its Structural Analogues. Macromolecules 2016, 49 (7), 2559-2567.

(11) Sakhawoth, Y.; Michot, L. J.; Levitz, P.; Malikova, N., Flocculation of Clay Colloids Induced by Model Polyelectrolytes: Effects of Relative Charge Density and Size. ChemPhysChem, n/a-n/a.

(12) Piradashvili, K.; Alexandrino, E. M.; Wurm, F. R.; Landfester, K., Reactions and Polymerizations at the Liquid-Liquid Interface. Chemical Reviews 2016, 116 (4), 2141-2169.

(13) Pramanik, S. K.; Seneca, S.; Ethirajan, A.; Neupane, S.; Renner, F. U.; Losada-Pérez, P., Ionic strength dependent vesicle adsorption and phase behavior of anionic phospholipids on a gold substrate. Biointerphases 2016, 11 (1), 019006.

(14) Israelachvili, J. N., 7 - Repulsive Steric Forces, Total Intermolecular Pair Potentials, and Liquid Structure. In Intermolecular and Surface Forces (Third Edition), Academic Press: San Diego, 2011; pp 133-149.

(15) Gebbie, M. A.; Valtiner, M.; Banquy, X.; Fox, E. T.; Henderson, W. A.; Israelachvili, J. N., Ionic liquids behave as dilute electrolyte solutions. Proceedings of the National Academy of Sciences 2013, 110 (24), 9674-9679.

(16) Perkin, S.; Salanne, M.; Madden, P.; Lynden-Bell, R., Is a Stern and diffuse layer model appropriate to ionic liquids at surfaces? Proceedings of the National Academy of Sciences of the United States of America 2013, 110 (44), E4121-E4121.

(17) Baimpos, T.; Shrestha, B. R.; Raman, S.; Valtiner, M., Effect of Interfacial lon Structuring on Range and Magnitude of Electric Double Layer, Hydration, and Adhesive Interactions between Mica Surfaces in 0.05-3 M Li+ and Cs+ Electrolyte Solutions. Langmuir 2014, 30 (15), 4322-4332.

(18) Lee, A. A.; Perez-Martinez, C. S.; Smith, A. M.; Perkin, S., Scaling Analysis of the Screening Length in Concentrated Electrolytes. Physical Review Letters 2017, 119 (2), 026002. 
(19) Vchirawongkwin, V.; Hofer, T. S.; Randolf, B. R.; Rode, B. M., TI(I)-the strongest structurebreaking metal ion in water? A quantum mechanical/molecular mechanical simulation study. Journal of Computational Chemistry 2007, 28 (6), 1006-1016.

(20) Marx, D.; Sprik, M.; Parrinello, M., Ab initio molecular dynamics of ion solvation. The case of Be2+ in water. Chemical Physics Letters 1997, 273 (5), 360-366.

(21) Bernal - Uruchurtu, M. I.; Ortega - Blake, I., A refined Monte Carlo study of Mg2+ and Ca2+ hydration. The Journal of Chemical Physics 1995, 103 (4), 1588-1598.

(22) Lightstone, F. C.; Schwegler, E.; Hood, R. Q.; Gygi, F.; Galli, G., A first principles molecular dynamics simulation of the hydrated magnesium ion. Chemical Physics Letters 2001, 343 (5-6), 549555.

(23) Tongraar, A.; Liedl, K. R.; Rode, B. M., Solvation of Ca2+ in Water Studied by Born-Oppenheimer ab Initio QM/MM Dynamics. The Journal of Physical Chemistry A 1997, 101 (35), 6299-6309.

(24) Rasaiah, J. C.; Lynden-Bell, R. M., Computer simulation studies of the structure and dynamics of ions and non-polar solutes in water. Philosophical Transactions of the Royal Society of London. Series A: Mathematical, Physical and Engineering Sciences 2001, 359 (1785), 1545.

(25) Du, H.; Rasaiah, J. C.; Miller, J. D., Structural and Dynamic Properties of Concentrated Alkali Halide Solutions: A Molecular Dynamics Simulation Study. The Journal of Physical Chemistry B 2007, $111(1), 209-217$.

(26) Israelachvili, J. N., 15 - Solvation, Structural, and Hydration Forces. In Intermolecular and Surface Forces (Third Edition), Academic Press: San Diego, 2011; pp 341-380.

(27) Derjaguin, B.; Landau, L., Theory of the stability of strongly charged lyophobic sols and of the adhesion of strongly charged particles in solutions of electrolytes. Progress in Surface Science 1993, 43 (1), 30-59.

(28) Ducker, W. A.; Senden, T. J.; Pashley, R. M., Direct measurement of colloidal forces using an atomic force microscope. Nature 1991, 353 (6341), 239-241.

(29) Pashley, R. M., Hydration forces between mica surfaces in aqueous electrolyte solutions. Journal of Colloid and Interface Science 1981, 80 (1), 153-162.

(30) Pashley, R. M.; Quirk, J. P., The effect of cation valency on DLVO and hydration forces between macroscopic sheets of muscovite mica in relation to clay swelling. Colloids and Surfaces 1984, 9 (1), 1-17.

(31) Pashley, R. M., DLVO and hydration forces between mica surfaces in $\mathrm{Li}+, \mathrm{Na}+, \mathrm{K}+$, and $\mathrm{Cs}+$ electrolyte solutions: A correlation of double-layer and hydration forces with surface cation exchange properties. Journal of Colloid and Interface Science 1981, 83 (2), 531-546.

(32) Bourg, I. C.; Lee, S. S.; Fenter, P.; Tournassat, C., Stern Layer Structure and Energetics at MicaWater Interfaces. The Journal of Physical Chemistry C 2017, 121 (17), 9402-9412.

(33) Kasuya, M.; Sogawa, T.; Masuda, T.; Kamijo, T.; Uosaki, K.; Kurihara, K., Anion Adsorption on Gold Electrodes Studied by Electrochemical Surface Forces Measurement. The Journal of Physical Chemistry C 2016, 120 (29), 15986-15992.

(34) Israelachvili, J.; Wennerstrom, H., Role of hydration and water structure in biological and colloidal interactions. Nature 1996, 379 (6562), 219-225.

(35) Cheng, L.; Fenter, P.; Nagy, K. L.; Schlegel, M. L.; Sturchio, N. C., Molecular-Scale Density Oscillations in Water Adjacent to a Mica Surface. Physical Review Letters 2001, 87 (15), 156103.

(36) Grahame, D. C., The Electrical Double Layer and the Theory of Electrocapillarity. Chemical Reviews 1947, 41 (3), 441-501.

(37) Kaspar, J., Zur Theorie der elektrolytischen Doppelschicht und der potentialbestimmenden Ionenadsorption. Helvetica Chimica Acta 1938, 21 (1), 650-663.

(38) Parametric tip model and force-distance relation for Hamaker constant determination from atomic force microscopy. Journal of Applied Physics 1996, 80 (11), 6081-6090.

(39) Israelachvili, J. N., Adams, G.E., Measurement of forces between 2 mica surfaces in aqueous electrolyte solutions in range 0-100 nm. . Journal of the Chemical Society, Faraday Transactions 1978, 74 (1), 975-1001. 
(40) Valtiner, M.; Kristiansen, K.; Greene, G. W.; Israelachvili, J. N., Effect of Surface Roughness and Electrostatic Surface Potentials on Forces Between Dissimilar Surfaces in Aqueous Solution. Advanced Materials 2011, 23 (20), 2294-2299.

(41) Israelachvili, J. N., Chapter 13 - Van der Waals Forces between Particles and Surfaces. In Intermolecular and Surface Forces (Third Edition), Academic Press: San Diego, 2011; pp 253-289.

(42) Parker, J. L., A novel method for measuring the force between two surfaces in a surface force apparatus. Langmuir 1992, 8 (2), 551-556.

(43) Butt, H.-J., Electrostatic interaction in atomic force microscopy. Biophysical Journal 1991, 60 (4), 777-785.

(44) Martin-Jimenez, D.; Chacon, E.; Tarazona, P.; Garcia, R., Atomically resolved three-dimensional structures of electrolyte aqueous solutions near a solid surface. Nature Communications 2016, 7, 12164

(45) P.V. Kiely, M. L. J., Selective dissolution of micas from potassium feldspars by sodium pyrosulfate fusion of soils and sediments. The amercian mineralogist 1964, 49.

(46) Toney, M. F.; Howard, J. N.; Richer, J.; Borges, G. L.; Gordon, J. G.; Melroy, O. R.; Wiesler, D. G.; Yee, D.; Sorensen, L. B., Voltage-dependent ordering of water molecules at an electrode-electrolyte interface. Nature 1994, 368 (6470), 444-446.

(47) Park, S.-H.; Sposito, G., Structure of Water Adsorbed on a Mica Surface. Physical Review Letters 2002, 89 (8), 085501.

(48) Xu, D.; Leng, Y.; Chen, Y.; Li, D., Water structures near charged (100) and (111) silicon surfaces. Applied Physics Letters 2009, 94 (20), 201901.

(49) Yinghua Qiu, Q. T., Weichuan Guo, Yunfei Chen, The Effects of lons and Surface Charge Density on Water Distribution in Silicon Nanochannel. In ASME 2012 International Mechanical Engineering Congress and Exposition, Houston USA, 2012.

(50) Gaines, G. L.; Rutkowski, C. P., The Extraction of Aluminum and Silicon from Muscovite Mica by Aqueous Solutions. The Journal of Physical Chemistry 1957, 61 (10), 1439-1441.

(51) Kristiansen, K.; Valtiner, M.; Greene, G. W.; Boles, J. R.; Israelachvili, J. N., Pressure solution - The importance of the electrochemical surface potentials. Geochimica Et Cosmochimica Acta 2011, 75 (22), 6882-6892.

(52) Hofmeister, F., Zur Lehre von der Wirkung der Salze. Archiv für experimentelle Pathologie und Pharmakologie 1888, 24 (4), 247-260.

(53) Schneider, J.; Dufrêne, Y. F.; Barger, W. R.; Lee, G. U., Atomic Force Microscope Image Contrast Mechanisms on Supported Lipid Bilayers. Biophysical Journal 2000, 79 (2), 1107-1118.

(54) Idziak, S. H. J.; Safinya, C. R.; Hill, R. S.; Kraiser, K. E.; Ruths, M.; Warriner, H. E.; Steinberg, S.; Liang, K. S.; Israelachvili, J. N., The X-ray Surface Forces Apparatus: Structure of a Thin Smectic Liquid Crystal Film Under Confinement. Science 1994, 264 (5167), 1915.

(55) Golan, Y.; Martin-Herranz, A.; Li, Y.; Safinya, C. R.; Israelachvili, J., Direct Observation of ShearInduced Orientational Phase Coexistence in a Lyotropic System Using a Modified X-Ray Surface Forces Apparatus. Physical Review Letters 2001, 86 (7), 1263-1266.

(56) Golan, Y.; Seitz, M.; Luo, C.; Martin-Herranz, A.; Yasa, M.; Li, Y.; Safinya, C. R.; Israelachvili, J., The $\mathrm{x}$-ray surface forces apparatus for simultaneous $\mathrm{x}$-ray diffraction and direct normal and lateral force measurements. Review of Scientific Instruments 2002, 73 (6), 2486-2488.

(57) Ueber die Berührung fester elastischer Körper. In Journal für die reine und angewandte Mathematik (Crelle's Journal), 1882; Vol. 1882, p 156.

(58) Marcus, Y., Thermodynamics of solvation of ions. Part 5.-Gibbs free energy of hydration at 298.15 K. Journal of the Chemical Society, Faraday Transactions 1991, 87 (18), 2995-2999.

(59) Marcus, Y., lonic radii in aqueous solutions. Chemical Reviews 1988, 88 (8), 1475-1498. 


\section{FIGURES AND TABLES.}

Figure 1: Schematic of the set-up used for probing interfacial ion structures by measuring interaction forces $\mathrm{F}$ during approach of an AFM tip and a mica surface. At $\mathrm{pH} \sim 1$ both mica and tip surfaces are positively charged through protonation. On the right side shows the distance dependent variation of the ion concentration $\Delta \mathrm{c}$ within the double layer is compared to the bulk value $\left(\mathrm{c}_{(\text {bulk })}\right)$. Near a positively charged surface, there is an accumulation of the anions. Due to the hydration shells around they can impose steric hydration forces during interaction of tip and surface.

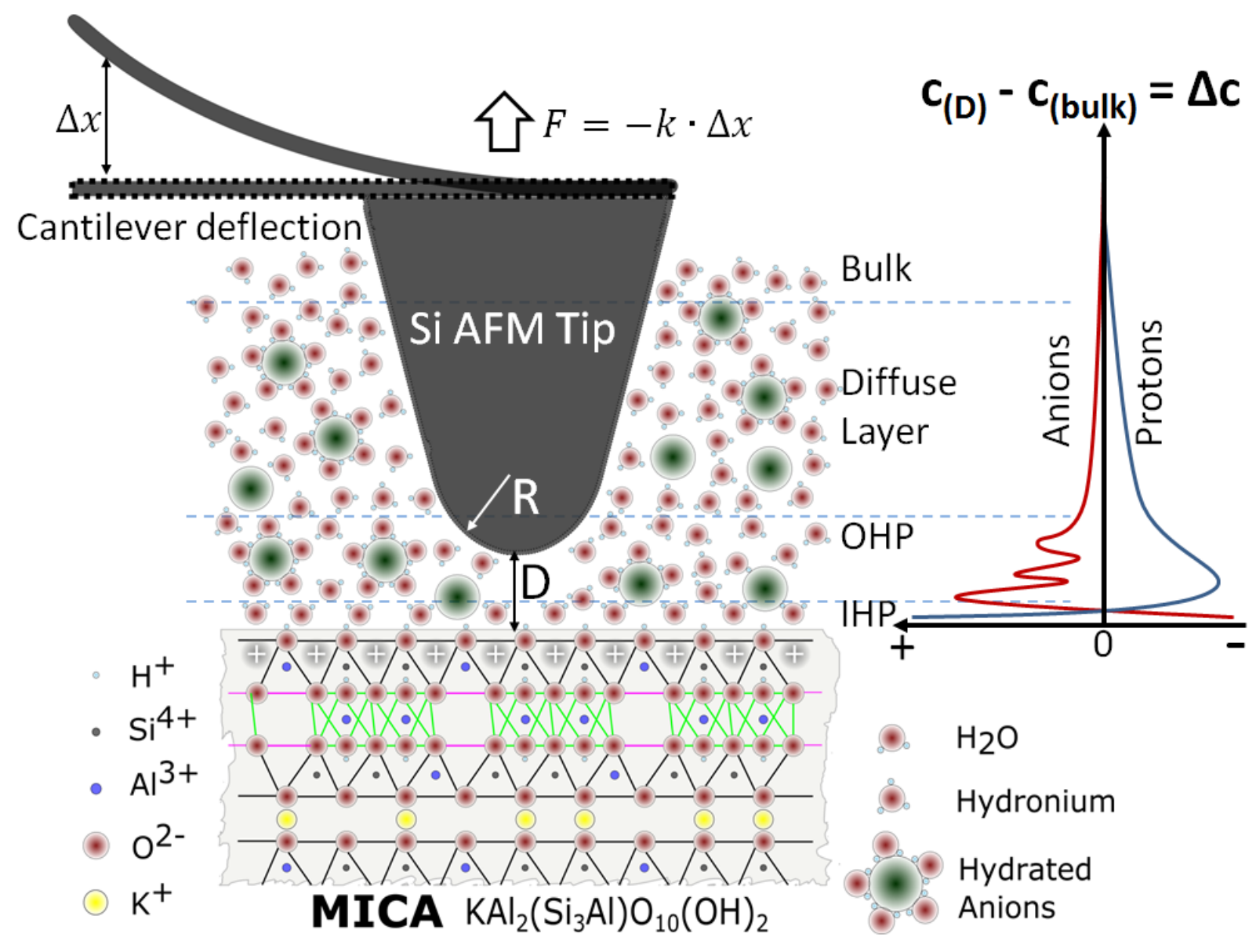


Figure 2: Interaction forces measured with AFM in non-degassed Milli-Q water ( $\mathrm{pH}$ 5.5) between a Si tip and a mica surface during approach. (a) Semi-logarithmic plot of a typical F-D curve during approach, solid curves are the fittings using Equation 4 and EDL force from work of H.-J. Butt ${ }^{43}$, (b) 2-D force versus distance heat-plot and zoom-in. Inset: Histogram of the force versus distance data.
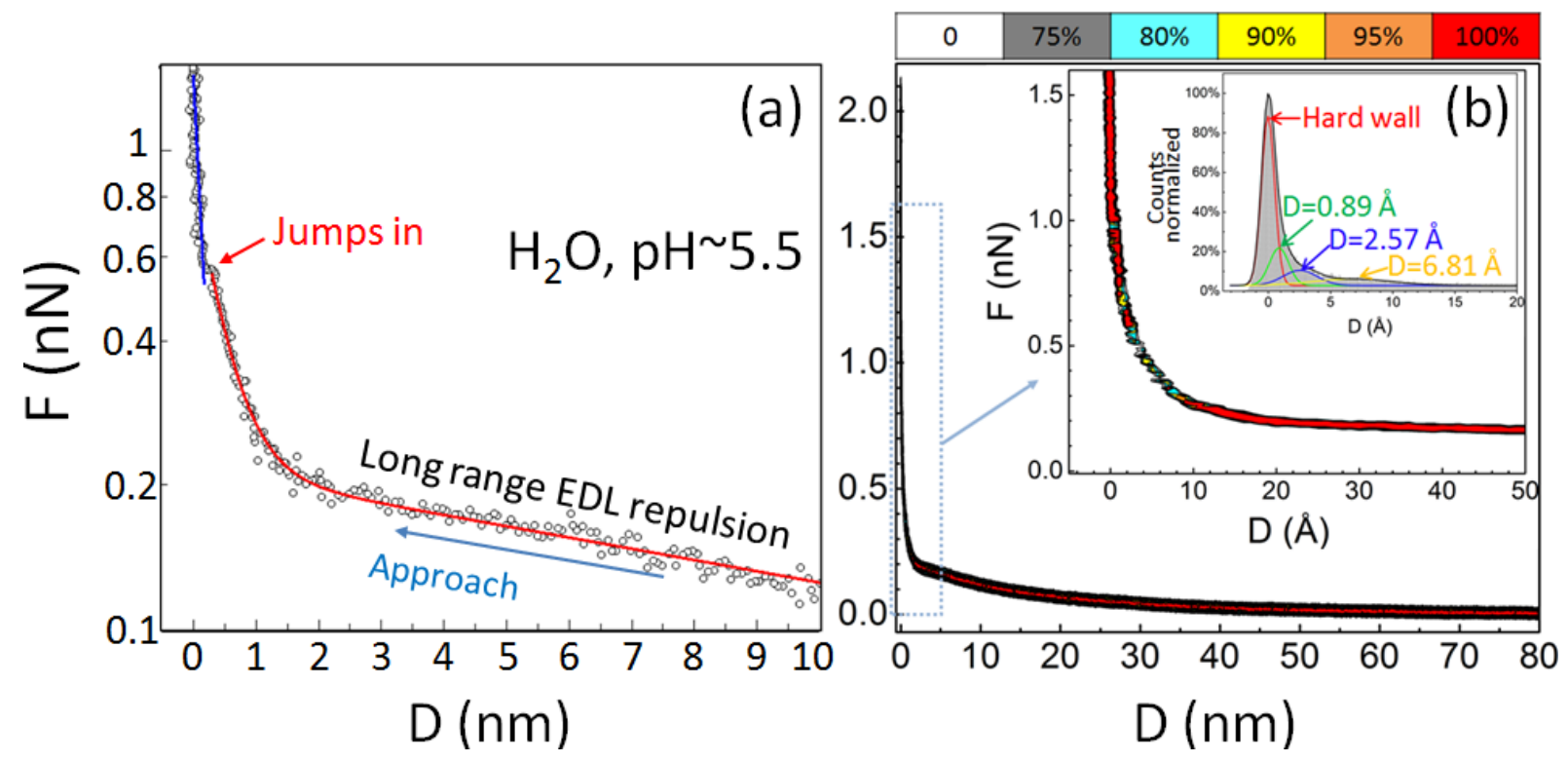

Figure 3: Schematic interpretation of the water structure at the interface of the negatively charged mica surface at $\mathrm{pH} \sim 5.5$. One strongly structured layer at $1 \AA$, and an instability corresponding roughly to the size of a water molecule at $2.5 \AA$, and diffuse structuring over two further water layers up to about $\mathrm{D}=9 \AA$ \& was observed.

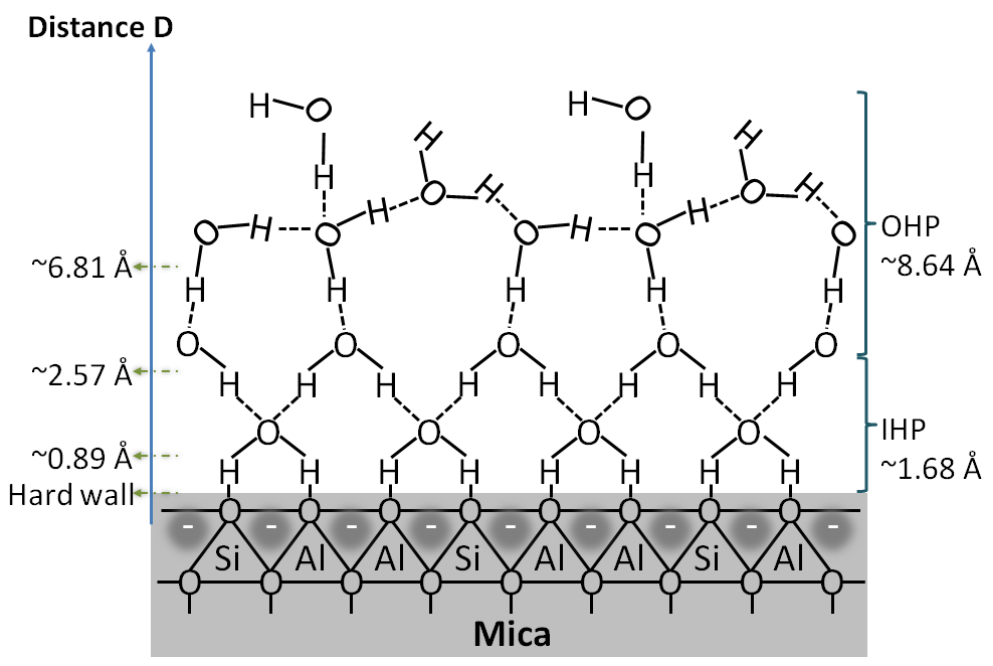

Figure 4: Comparison of 2-D interaction forces at the distance below $50 \AA$ measured with AFM between Si tip and mica surface during approach in different acidic $100 \mathrm{mM}$ electrolytes: (a) $\mathrm{HCl}$, (b) 
$\mathrm{HClO}_{4}$, (c) $\mathrm{HNO}_{3}$, (d) $\mathrm{H}_{2} \mathrm{SO}_{4}$ and (e) $\mathrm{H}_{3} \mathrm{PO}_{4}$. The color scale is similar compared to Figure 2, only showing probabilities over $75 \%$. Insets plot all the force histograms over distances within $2 \mathrm{~nm}$.

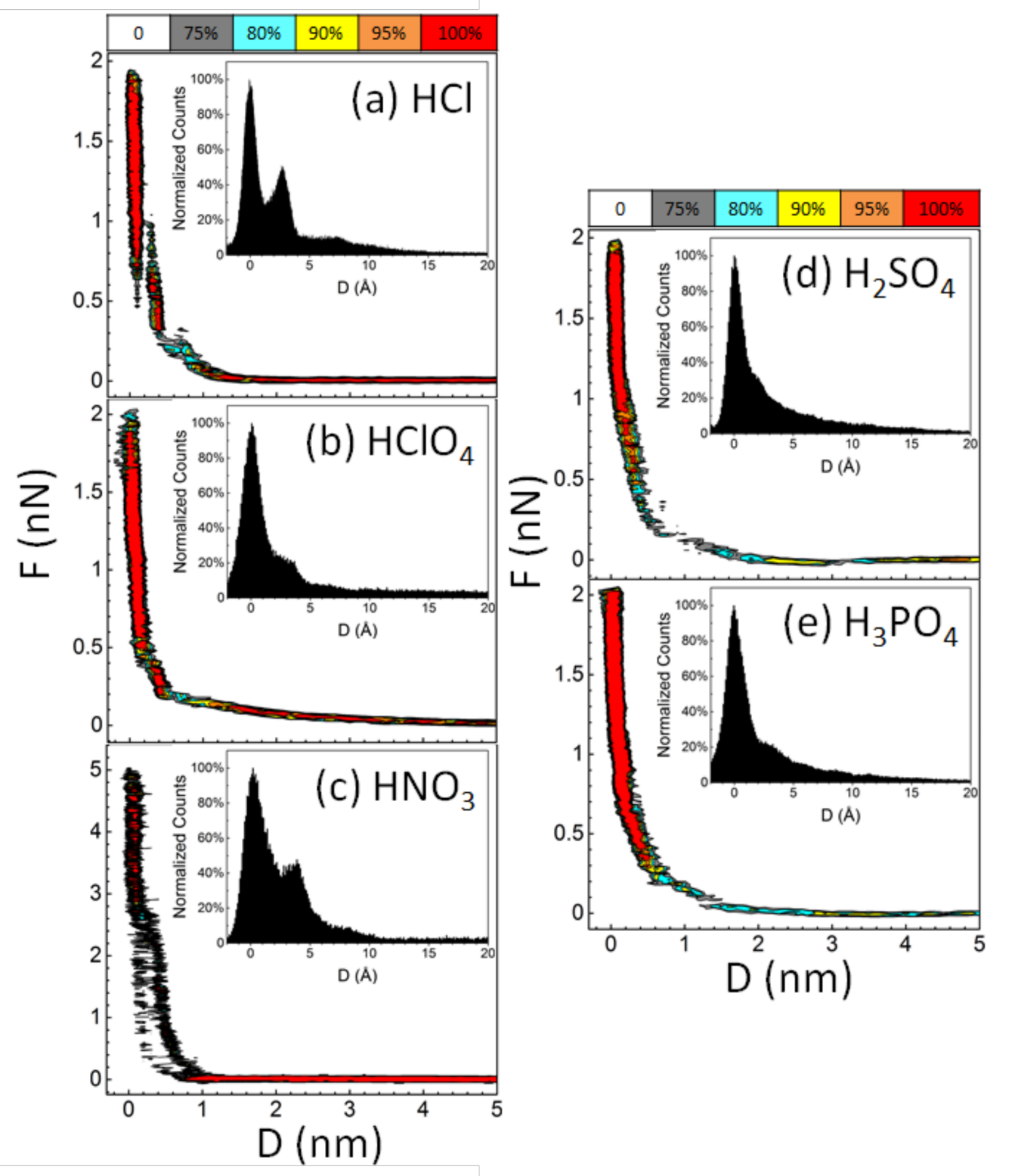

Figure 5: Fitting of individual F-D curves using Equation 4, indicating a good empirical description of the force distance profiles using only the radius $\mathrm{R}$, the decay lengths and the exponential prefactros $\mathrm{W}_{0}$ as fitting variables. As can be seen immediately, the mica surface charge is screened within 1-3 nm 
in acidic solutions, while $50 \%$ of the mica surface charge decays over the Debye length of $38 \mathrm{~nm}$ in water at $\mathrm{pH}=5.5$ (cf. text for details).
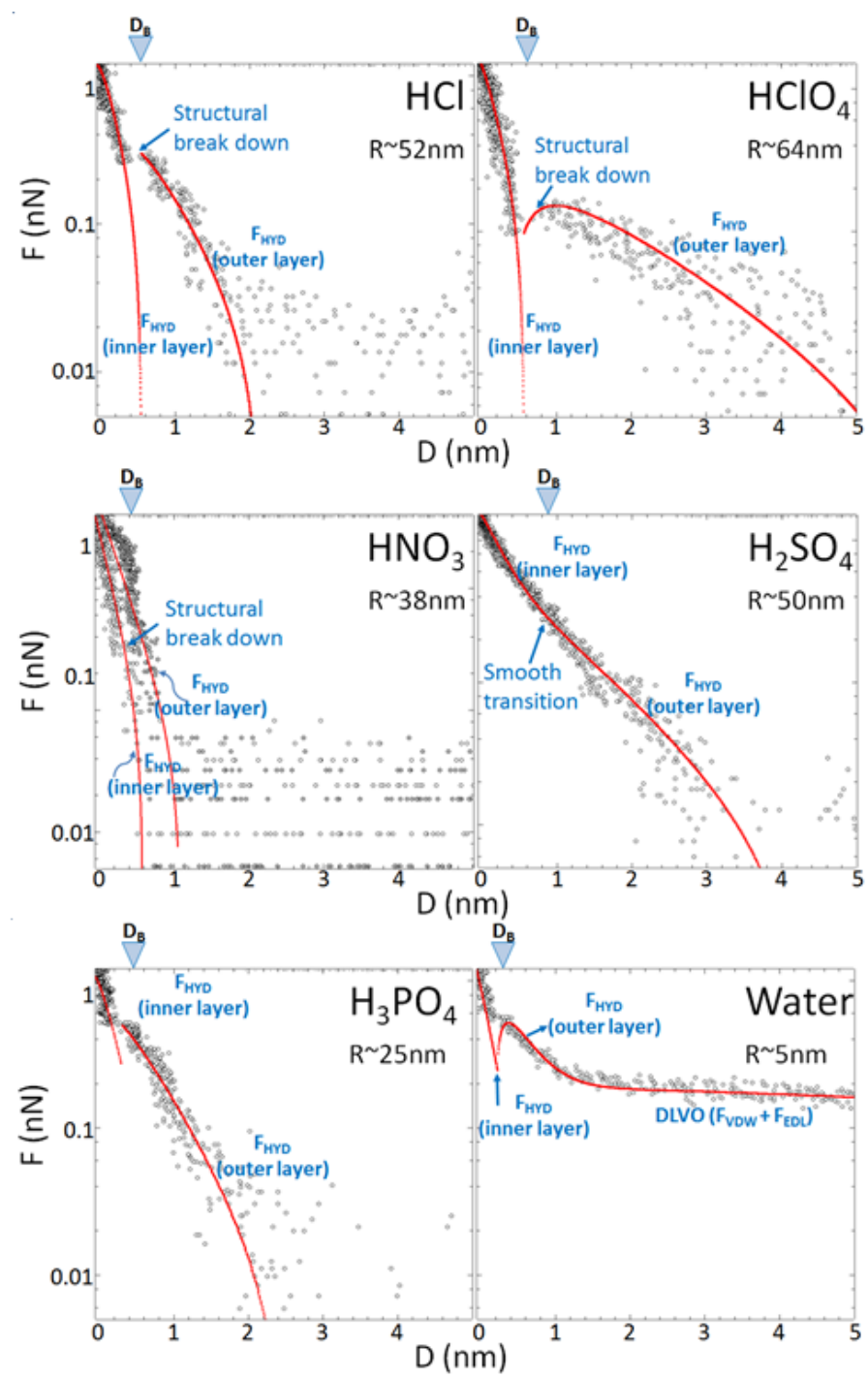

Figure 6: Adhesive forces and adhesive energy per unit area calculated from Hertz contact model ${ }^{57}$. 


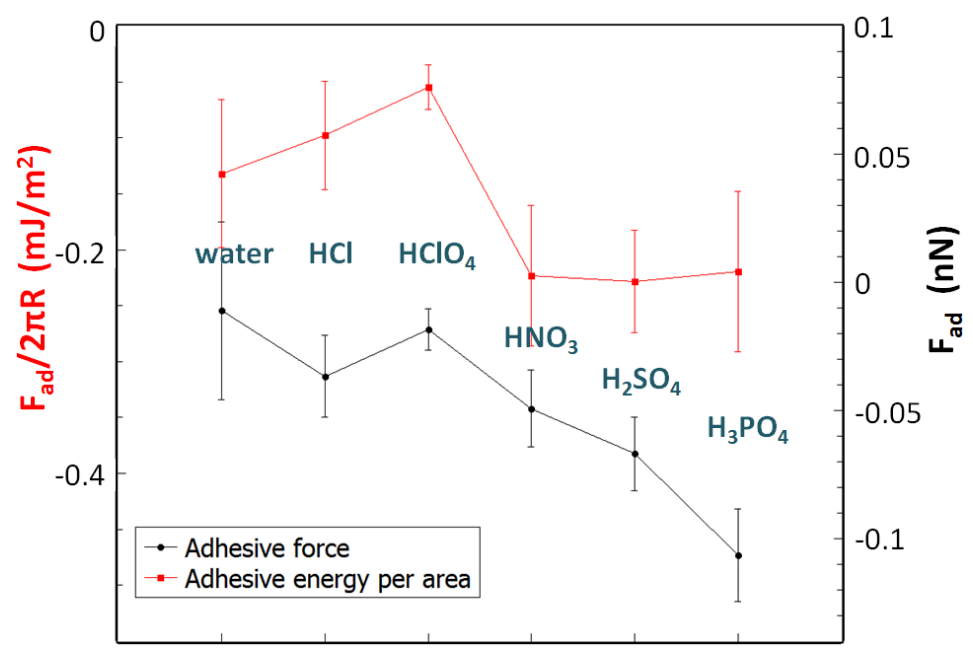

Table 1: The diameter of hydrated ions $d_{\text {hyd }}$, hydration enthalpy and entropy values, molar free energies of hydration $\left(\Delta G_{h y d}\right)$ at room temperature, as well as lyotropic numbers $N_{\text {lyo }}$ are quoted from literature ${ }^{58,59}$.

\begin{tabular}{cccccc}
\hline lons & $\begin{array}{c}\mathbf{d}_{\text {hyd }} \\
(\AA)\end{array}$ & $\begin{array}{c}\Delta \mathrm{H}_{\text {hyd }} \\
(\mathbf{k J} / \mathrm{mol})\end{array}$ & $\begin{array}{c}\Delta \mathrm{S}_{\text {hyd }} \\
(\mathbf{k J} / \mathrm{mol})\end{array}$ & $\begin{array}{c}\Delta \mathbf{G}_{\text {hyd }} \\
(\mathbf{k J} / \mathrm{mol})\end{array}$ & $\mathbf{N}_{\text {lyo }}$ \\
\hline $\mathbf{H}^{+}$ & - & -1103 & -131 & -1064 & - \\
\hline $\mathrm{Cl}^{-}$ & 6.4 & -367 & -76 & -344 & 10 \\
$\mathrm{ClO}_{4}{ }^{-}$ & $7.0-7.6$ & -246 & -57 & -229 & 11.8 \\
$\mathrm{NO}_{3}{ }^{-}$ & equatorial: 5.3 & -312 & -86 & -286 & 11.6 \\
$\mathbf{H S O}_{4}^{-}$ & axial: 6.9 & & & & \\
$\mathbf{S O}_{4}{ }^{2-}$ & 7.6 & -368 & -129 & -330 & \\
$\mathbf{H}_{2} \mathbf{P O}_{4}{ }^{-}$ & 7.2 & -1035 & -200 & -975 & 2.0 \\
\hline
\end{tabular}


Table 2: Relevant extended fitting parameters for hydration forces as described in $4 . \mathrm{D}_{\mathrm{T}}$ is the distance at which the outer layer transitions into the inner hydration layer. The force at this distance, is equivalent for the Debye length, the expected values are given in brackets in the left column.

\begin{tabular}{|c|c|c|c|c|c|c|}
\hline \multirow{2}{*}{$\begin{array}{c}\text { Electrolyte } \\
\left.\text { (expected } \lambda_{D}\right)\end{array}$} & \multirow{2}{*}{$\begin{array}{l}\text { Radius } \\
\text { (nm) }\end{array}$} & \multicolumn{2}{|c|}{$F_{H Y D}$ (inner layer) } & \multicolumn{2}{|c|}{ Transition } & \multirow{2}{*}{ 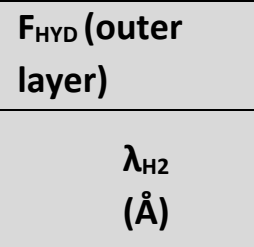 } \\
\hline & & $\begin{array}{c}2 \pi R W_{01} \\
(n N)\end{array}$ & $\begin{array}{l}\lambda_{\mathrm{H} 1} \\
\text { (Å) }\end{array}$ & $\begin{array}{l}\mathrm{D}_{\mathrm{T}} \\
(\AA \AA)\end{array}$ & $\begin{array}{c}\mathrm{F} @ \mathrm{D}_{\mathrm{T}} \\
(\mathrm{nN})\end{array}$ & \\
\hline \multicolumn{7}{|l|}{ Milli Q-water } \\
\hline $\begin{array}{l}\text { pH } 5.5 \\
\text { (50 Å) }\end{array}$ & 5.0 & 1.76 & $1.55 \pm 0.25$ & 2.95 & 0.55 & $3.35 \pm 0.35$ \\
\hline $\begin{array}{c}\mathrm{HCl} \\
(9.6 \AA \AA)\end{array}$ & 52 & 2.86 & $2.4 \pm 0.2$ & 6.39 & 0.27 & $6 \pm 0.5$ \\
\hline $\begin{array}{l}\mathrm{HClO}_{4} \\
(9.6 \AA)\end{array}$ & 64 & 3.42 & $2.45 \pm 0.25$ & 9.53 & 0.14 & $13.5 \pm 0.5$ \\
\hline $\begin{array}{l}\mathrm{HNO}_{3} \\
(9.6 \AA)\end{array}$ & 38 & 3.33 & $1.8 \pm 0.2$ & \multicolumn{2}{|c|}{$\begin{array}{l}\text { independent } \\
\text { layers }\end{array}$} & $2.45 \pm 0.25$ \\
\hline $\begin{array}{l}\mathrm{H}_{2} \mathrm{SO}_{4} \\
(8.9 \AA)\end{array}$ & 25 & 0.88 & $2.5 \pm 0.25$ & 8.24 & 0.33 & $8 \pm 0.15$ \\
\hline $\begin{array}{l}\mathrm{H}_{3} \mathrm{PO}_{4} \\
(9.6 \AA)\end{array}$ & 25 & 1.83 & $2.5 \pm 0.3$ & 4.00 & 0.47 & $5.6 \pm 0.4$ \\
\hline
\end{tabular}

